\title{
Identification of an Amygdala-Thalamic Circuit That Acts as a Central Gain Mechanism in Taste Perceptions
}

\author{
Maria G. Veldhuizen, ${ }^{7}$ Michael C. Farruggia, ${ }^{1,2,3}$ Xiao Gao, ${ }^{8,9}$ Yuko Nakamura, ${ }^{10}$ Barry G. Green, ${ }^{5,6}$ and \\ Dana M. Small ${ }^{1,2,3,4}$ \\ ${ }^{1}$ Department of Psychiatry, Yale School of Medicine, New Haven, Connecticut 06511, ${ }^{2}$ Modern Diet and Physiology Research Laboratory, Yale \\ School of Medicine, New Haven, Connecticut 06519, ${ }^{3}$ Interdepartmental Neuroscience Program, Yale University, New Haven, Connecticut 06510, \\ ${ }^{4}$ Department of Psychology, Yale University, New Haven, Connecticut 06510, ${ }^{5}$ The John B. Pierce Laboratory, New Haven, Connecticut 06519, \\ ${ }^{6}$ Department of Surgery (Otolaryngology), Yale School of Medicine, New Haven, Connecticut 06510, ${ }^{7}$ Department of Anatomy, Faculty of Medicine, \\ Mersin University, Ciftlikkoy Campus, 33343, Mersin, Turkey, ${ }^{8}$ Faculty of Psychology, Southwest University, Chongqing, 400715, China, ${ }^{9}$ Key \\ Laboratory of Cognition and Personality, Southwest University, Chongqing, China, and ${ }^{10}$ The UTokyo Center for Integrative Science of Human \\ Behavior, Tokyo, Japan
}

Peripheral sources of individual variation in taste intensity perception have been well described. The existence of a central source has been proposed but remains unexplored. Here we used functional magnetic resonance imaging in healthy human participants ( 20 women, $8 \mathrm{men}$ ) to evaluate the hypothesis that the amygdala exerts an inhibitory influence that affects the "gain" of the gustatory system during tasting. Consistent with the existence of a central gain mechanism (CGM), we found that central amygdala response was correlated with mean intensity ratings across multiple tastants. In addition, psychophysiological and dynamic causal modeling analyses revealed that the connection strength between inhibitory outputs from amygdala to medial dorsal and ventral posterior medial thalamus predicted individual differences in responsiveness to taste stimulation. These results imply that inhibitory inputs from the amygdala to the thalamus act as a CGM that influences taste intensity perception.

Key words: amygdala; fMRI; gustation; perception; taste intensity; thalamus

\section{Significance Statement}

Whether central circuits contribute to individual variation in taste intensity perception is unknown. Here we used functional magnetic resonance imaging in healthy human participants to identify an amygdala-thalamic circuit where network dynamics and connectivity strengths during tasting predict individual variation in taste intensity ratings. This finding implies that individual differences in taste intensity perception do not arise solely from variation in peripheral gustatory factors.

\section{Introduction}

Taste acts as a gatekeeper to our internal milieu, with taste quality tuned to identify potentially nutritive or harmful substances (Breslin, 2013) and taste intensity reflecting concentration, and hence quantity, of these substances. Notably, there are considerable individual differences in taste intensity perception that investigators have endeavored to link with food preferences, diet, and risk for obesity and alcohol consumption (Duffy and

\footnotetext{
Received Nov. 4, 2019; revised Feb. 10, 2020; accepted Mar. 19, 2020.

Author contributions: B.G.G. and D.M.S. designed research; M.G.V., X.G., Y.N., and D.M.S. performed research; M.V., M.C.F., X.G., and D.M.S. analyzed data; D.M.S. wrote the paper

The authors declare no competing financial interests.

This work was supported by National Institutes of Health Training Grant T32-NS-041228 (M.C.F.); the Yale Medical School Fellowship (M.C.F.); and National Institutes of Health Grant R01-DC-006706 (D.M.S.).

Correspondence should be addressed to Maria G. Veldhuizen at margaveldhuizen@gmail.com or Dana M. Small at dana.small@yale.edu.

https://doi.org/10.1523/JNEUROSCI.2618-19.2020

Copyright $\odot 2020$ the authors
}

Bartoshuk, 2000; Pepino and Mennella, 2007; Hayes et al., 2011; Allen et al., 2014; Thibodeau et al., 2017). Investigations into the source of this variance have ocused primarily on possible peripheral mechanisms, such as allelic variation in the TAS2R38 bitter taste receptor gene or the number of taste papillae, but the evidence for such mechanisms in humans is not strong (for review, see Feeney and Hayes, 2014). Less well explored is the possible influence of central mechanisms. However, there is evidence that such a mechanism may exist. Studies of the phenomenon of thermal taste, in which some individuals perceive taste sensations from temperature alone (Cruz and Green, 2000), has shown that the ability to perceive thermal taste is significantly correlated with ratings of the perceived intensity of chemical tastes and the sweet odorant vanillin (Green and George, 2004; Green et al., 2005; Lim et al., 2008). Importantly, the same studies also showed that the individuals who perceived thermal taste did not rate oral somatosensory sensations of creaminess, spiciness, and astringency or thermal sensations produced on the lip and hand 
any higher than individuals who did not perceive thermal taste. The specificity of these correlations to taste and olfactory stimuli rules out a cognitive bias or differences in scale usage as significant factors in the individual differences. Furthermore, because taste, olfaction, and somesthetic stimulation (temperature, creaminess, and chemical irritation) are all transduced by different peripheral receptors and cranial nerves, these data suggest that the correlations are driven primarily by a central neural mechanism that is common to taste and smell but not to somesthesis (Green and George, 2004; Green et al., 2005).

The neurobiology of such a "central gain mechanism" (CGM) is unexplored. However, early evidence of taste perception in patients who had undergone surgical resection of the anterior medial temporal lobe for the treatment of pharmacologically intractable epilepsy suggests amygdala involvement. Specifically, resections involving the amygdala, particularly from the right hemisphere, result in reduced sour taste detection thresholds (i.e., increased sensitivity; Small et al., 1997) and stronger ratings of the perceived intensity of suprathreshold taste stimuli (Small et al., 2001a,b). These paradoxical observations have been interpreted as evidence for an inhibitory influence from the amygdala to either the gustatory cortex in the insula, or to earlier taste relays (Small et al., 2001b). Blood oxygenation level-dependent (BOLD) responses in the central nucleus are sensitive to modulation of intensity perception (Small et al., 2003), and there are reciprocal connections between the amygdala and the insular taste cortex (Wang et al., 2018), the gustatory thalamus [ventral posterior medial nucleus (VPM); Krettek and Price, 1977], and brainstem [rostral nucleus of the solitary tract (NST); Norgren, 1976] relays. In addition, the amygdala has previously been described as conferring gain during directed attention by biasing signals to modulate early sensory cortex, assigning greater "weights" to emotionally relevant or salient sensory events (Vuilleumier, 2015).

The current study was designed to test the hypothesis that individual variation in taste intensity perception is associated with inhibitory projections from the amygdala to gustatory regions. Our approach was to assess BOLD response to sweet, sour, salty, and bitter tastants in 28 healthy participants using regression, psychophysiological measurement, and dynamic causal modeling (DCM) analyses to determine whether (1) amygdala response to the tastants covaried with the mean intensity rating across tastants; (2) the responsive region of the amygdala was functionally coupled to gustatory regions including brainstem, thalamus, and insula; and (3) using Bayesian model estimation, whether inhibitory influences from the amygdala to gustatory regions (i.e., effective connectivity; Friston et al., 2011) characterize gustatory network dynamics associated with intensity perception.

\section{Materials and Methods \\ Participants}

Thirty right-handed, nonsmoking participants [21 women, 9 men; mean age, $27.53 \pm 4.99$ years; age range, $18-38$ years; mean body mass index (BMI), $21.67 \pm 2.88 \mathrm{~kg} / \mathrm{m}^{2}$; BMI range, $16.08-26.93$ $\mathrm{kg} / \mathrm{m}^{2}$ ], with a mean Edinburgh Handedness Inventory (Oldfield, 1971) score of $90.17 \pm 11.41$, participated in the current study. They were recruited through advertisements around Yale University and the city of New Haven. The Yale School of Medicine Human Investigation Committee approved the informed consent form, which was subsequently obtained from all study participants. All participants reported having no known taste, smell, neurologic, psychiatric, or other pathologic disorders. Two (of the original 30) participants were excluded because one participant's average taste intensity rating was $<2$ SDs from the sample mean and another participant's head movements during scanning exceeded a predetermined limit of $1 \mathrm{~mm}$ of movement in any direction. The remaining 28 participants (20 women and 8 men) were a mean age of $27.14 \pm 4.75$ years with an age range of $18-37$ years, and had a mean BMI of $21.58 \pm 2.96 \mathrm{~kg} / \mathrm{m}^{2}$ with a range of $16.08-26.93 \mathrm{~kg} / \mathrm{m}^{2}$.

\section{Taste stimuli and delivery}

The taste stimuli consisted of a sweet $[0.32 \mathrm{~m}$ sucrose (Su), SigmaAldrich], sour $\left(5.6 \times 10^{-3} \mathrm{M}\right.$ citric acid (CA)], salty [0.14 M sodium chloride $(\mathrm{NaCl})]$, bitter $\left[3.2 \times 10^{-5} \mathrm{M}\right.$ quinine sulfate $\left.(\mathrm{Qu})\right]$, and a "tasteless" solution designed to mimic the ionic components of saliva (O'Doherty et al., 2001). We used the tasteless solution as the control stimulus because water activates taste cortex (Frey and Petrides, 1999; Zald and Pardo, 2000) and is sometimes reported to have a taste (Bartoshuk et al., 1964). The tasteless solution was mixed to the following five different concentrations: $18.75 \mathrm{~mm} \mathrm{KCl} / 1.875 \mathrm{~mm} \mathrm{NaHCO} 3$ in distilled water, and its $75 \%, 50 \%, 25 \%$, and $12.5 \%$ dilutions. Liquids were delivered to the participants through a portable gustometer system as $0.5 \mathrm{ml}$ of solution infused into the mouth over $4 \mathrm{~s}$. Detailed description of the gustometer system can be found in previous publications (Veldhuizen et al., 2007, 2011b)

\section{Experimental procedures}

Participants took part in one training and taste psychophysics session, and one fMRI scanning session. Sessions were conducted on separate days.

Training and taste psychophysics session. This session served to select an appropriate tasteless solution for each participant; assess reliable intensity ratings of four tastants representing sweet, sour, salty, and bitter qualities; familiarize participants with the fMRI task' and identify those who found it uncomfortable to perform the task in a simulated fMRI environment. Participants were instructed to refrain from eating or drinking anything other than water for at least an hour before the session. Upon arrival, they received a detailed explanation of the study. After participants read and signed the consent form, anthropometric measurements were taken. Body weight was measured to the nearest $0.1 \mathrm{~kg}$ using a calibrated Seca mBCA 514 medical Body Composition Analyzer (Itin Scale Company) with light clothes on only, and height was measured to the nearest $0.1 \mathrm{~cm}$ using a calibrated Seca Digital stationary stadiometer (Itin Scale Company). Body mass index (BMI) was calculated as weight (in kilograms) divided by the squared height (in meters) of the participant $\left(\mathrm{BMI}=\mathrm{kg} / \mathrm{m}^{2}\right)$. Participants were then presented with the five versions of the tasteless solution and selected the one that "tastes most like nothing" in a sequential pairwise choice procedure.

Next, training on how to use the general labeled magnitude scale (gLMS) was provided. The gLMS is an experimentally derived scale validated for use in describing the breadth of intensities an individual can experience in daily life, from sights and sounds to tastes and pains (Green et al., 1993, 1996; Bartoshuk et al., 2004). The scale consists of a vertical line bounded by the labels "no sensation" at the bottom and "strongest imaginable sensation" at the top, with the intensity labels "barely detectable," "weak," "moderate," "strong," and "very strong" spaced quasi-logarithmically along the scale (Green et al., 1993). We then asked participants to use the gLMS, which was presented via computer, to rate the intensity of imagined stimuli, such as the sweetness of milk and the bitterness of fresh spring water as well as actual pure taste stimuli and taste stimuli in mixtures.

Following gLMS training, participants were brought to the fMRI simulator and outfitted with the taste delivery system. Participants lay supine within the simulated bore and were asked to rate the intensity of their feelings of hunger, fullness, thirst, anxiety, and need to urinate. Next, participants used the gLMS to rate the perceived intensity of the four taste stimuli and the tasteless solution they had selected. Each taste stimulus and tasteless solution was delivered eight times, resulting in 40 trials in total. The order of stimulus delivery was randomized across participants. In each trial (Fig. $1 A$ ), the participant was instructed to swallow immediately after receiving each liquid and then rate the intensity of the specific taste quality of that tastant (sweetness, saltiness, sourness, 
A

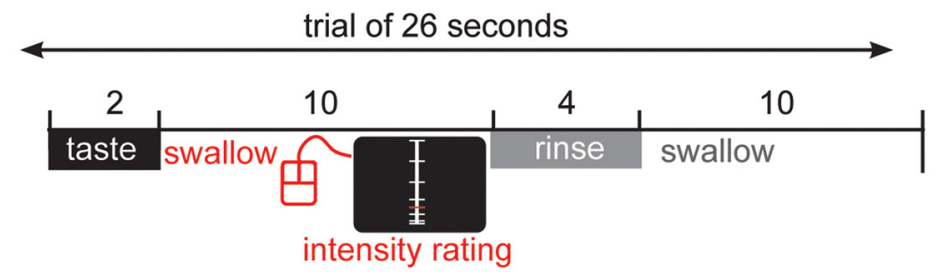

B
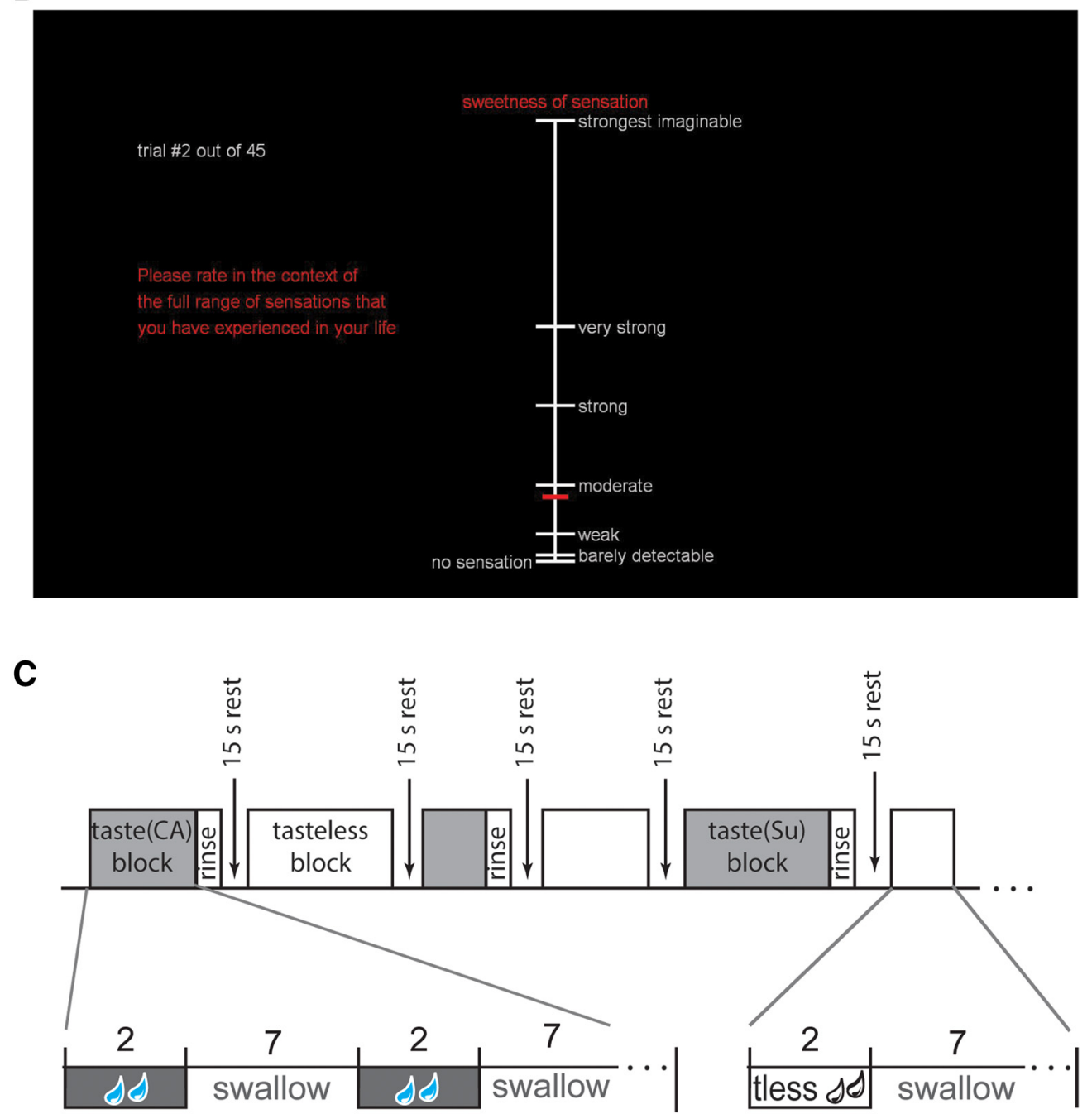

Figure 1. Overview of task design. $A$, Chronological events and their duration in a trial during the taste psychophysics session. Each trial commenced with a taste delivery of. $5 \mathrm{ml}$ over $2 \mathrm{~s}$. The participant was instructed to swallow immediately after receiving each liquid and then rate the intensity of taste. The intensity scale disappeared from the screen after $\sim 7 \mathrm{~s}$ or as soon as the rating was submitted by clicking a mouse button, whichever occurred first. Then a water rinse of $1 \mathrm{ml}$ was presented over $4 \mathrm{~s}$, followed by a rest period of $10 \mathrm{~s}$ before the next trial started, except after the tasteless stimulus, which was immediately followed by the next trial. $\boldsymbol{B}$, The gLMS that was used for measuring perceived taste intensity. The specific taste quality to be rated (e.g., "sweetness" for sucrose and "saltiness" for sodium chloride) was indicated above the scale. When a participant received the tasteless solution the label was "overall intensity," since no specific taste quality is associated with this stimulus. Participants used a scanner-compatible handheld trackball mouse to make ratings. The horizontal red cursor tracks up and down the scale with vertical mouse position, and clicking the left mouse button submitted the rating. A new gLMS then appeared on the screen to rate the next taste stimulus. $C$, Chronological events during a run in the fMRI session. Each block consisted of four, six, of eight repeats of a taste stimulus. Each repeat consists of a taste stimulus presentation over $2 \mathrm{~s}(0.5 \mathrm{ml})$, followed by a $7 \mathrm{~s}$ interval for swallowing. At the end of each block, a water rinse is presented over $4 \mathrm{~s}(1 \mathrm{ml})$, followed by a $15 \mathrm{~s}$ rest period before the start of the next block. Tasteless blocks are identical except that there is no water rinse before the $15 \mathrm{~s}$ rest period. Each run contained four taste blocks (one for each taste quality) and four tasteless blocks. No intensity ratings were made during the fMRI run.

bitterness; Fig. 1B). When they received the tasteless solution, they were asked to rate overall intensity, since there is no specific taste quality associated with this stimulus. Participants then made a second set of internal states. Liking was measured using a labeled hedonic scale (Lim et al., 2008) consisting of a vertical line scale with the labels "most disliked sensation imaginable" at the lower anchor point, "most liked sensation imaginable" at the upper anchor point, "neutral" in the middle, and "(dis)like slightly," "(dis)like moderately," "(dis)like very much," and "(dis)like extremely" spaced quasi-logarithmically along the scale. Familiarity and wanting were rated using two different visual analog scales. For wanting, "How much do you want to eat more of this?" ("I would never want to eat this" on the left anchor, "Neutral" in the middle, 
"I would want to eat this more than anything" on the right anchor); for familiarity, "How familiar is this taste?" ("Not familiar at all" on the left anchor, "Neutral" in the middle, "Very familiar" on the right anchor). All of the ratings were conducted in the bore of the simulator using a mouse on a computer monitor viewed via backprojection on a headcoilmounted mirror. Finally, participants underwent a simulation of a single scanner run, described in detail below.

fMRI scanning session. The fMRI scanning session took place on a separate day after the training and taste intensity rating session. Participants provided internal state ratings as well as perceptual ratings of the stimuli before and after scanning. Four taste runs were completed during this session. Each run was $9 \mathrm{~min}$ and $40 \mathrm{~s}$ long, and consisted of the uncued delivery of five different types of stimuli in eight blocks. Blocks had four, six, or eight repeats of the same stimulus. Each run consisted of one block for each of the four taste qualities [throughout this article referred to as taste $(\mathrm{Su})$, taste $(\mathrm{CA})$, taste $(\mathrm{NaCl})$, and taste $(\mathrm{Qu})]$ and four tasteless blocks. A tasteless block was coded as the tasteless control for the tastant that, within a given run, had the same number of repeats in a block [referred to as tasteless $(\mathrm{Su})$, tasteless $(\mathrm{CA})$, tasteless $(\mathrm{NaCl})$, and tasteless $(\mathrm{Qu})]$. In a block, a repeat of a stimulus presentation consisted of an "on" period of $3 \mathrm{~s}$, during which we presented $0.5 \mathrm{ml}$ of liquid, which was followed by a $6 \mathrm{~s}$ interval during which the subject is instructed to swallow (Fig. 1C). Following each block, there is a $1 \mathrm{ml}$ rinse with water followed by a $10 \mathrm{~s}$ rest. The participants were instructed to swallow after receiving each liquid and to keep their eyes closed during the entire run. The order of four taste stimuli blocks and four tasteless blocks within each run was counterbalanced, and the order of the runs was counterbalanced across participants. The number of repeats across taste blocks in this protocol was designed for collapsing across taste qualities and to provide balanced presentation of taste and tasteless blocks. Block design was chosen to improve power within participants and is favored for DCM analyses (Daunizeau et al., 2011).

fMRI scanner. Images were acquired on a Siemens TIM Trio 3T MRI Scanner equipped with a 32-channel head coil. Echoplanar imaging (EPI) was used to measure the BOLD signal as an indication of cerebral brain activation. A susceptibility-weighted echoplanar method with multiband acceleration factor 4 and GRAPPA (generalized, autocalibrating, partially parallel acquisitions) factor 2 was used to image the regional distribution of the BOLD signal with the following parameters: TR, $1000 \mathrm{~ms}$; TE, $30 \mathrm{~ms}$; flip angle, $60^{\circ}$; FOV, $220 \mathrm{~mm}$; matrix, $64 \times 64$; slice thickness, 2.5 $\mathrm{mm}$; number of slices, 60 . Slices were acquired in an interleaved mode, to reduce the cross talk of the slice selection pulse, and in coronal orientation, to cover the medulla oblongata of the brainstem, where the nucleus of the solitary tract is located. In total, 2376 volumes (594 volumes per run) were acquired. At the beginning of each functional run, the MR signal was allowed to equilibrate over two scans ("dummy images") for a total of $2 \mathrm{~s}$, which were then excluded from analysis. Anatomical scans were acquired with T1-weighted MPRAGE (magnetization-prepared rapid gradientecho sequence; TR/TE, 1900/2.52 ms; flip angle, $9^{\circ}$; FOV, 256; matrix, $256 \times 256$; slice thickness, $1 \mathrm{~mm}$; number of slices, 176). EPI-based field maps were also acquired to correct for susceptibility-induced geometric distortions.

\section{Data analysis}

Behavior. Taste intensity ratings made on the gLMS for each trial and each participant were first $\log _{10}$ transformed before statistical analysis. Eight intensity ratings for each of the tastants and the tasteless solution were then averaged within each participant, respectively. We ran a oneway repeated-measures ANOVA with GraphPad Prism version 7.01 for Windows to test for differences in average perceived intensity differences between the different stimuli. We performed paired $t$ tests with Bonferroni correction for 15 possible multiple comparisons. For each pair of different taste solutions (i.e., sweet-sour, sweet-salty sweet-bitter, sour-salty, sour-bitter, and salty-bitter) we then calculated the Pearson product-moment correlation of log-mean intensity ratings across participants. We performed one-tailed significance tests due to the strong a priori prediction of positive correlations between the intensities of the taste solutions and the absence of any rationale for a negative correlation. We then corrected for multiple comparisons using a Bonferroni correction for a total of six tests, leading to an $\alpha$ of 0.0167 . We averaged across the taste solutions that showed significant correlations in intensity to calculate a grand average score for each participant's taste intensity ratings. This included sweet, sour, and salty tastants, and excluded quinine as a tastant (see Taste psychophysics in the Results section). We used principal component analysis in $\mathrm{R}$ studio to confirm the existence of more than a single component in the variance across participants. We calculated a grand taste intensity score for each participant by averaging ratings for sweet, sour, and salty tastants, referred to hereafter as the mean $\log$ intensity ${ }^{\mu(\mathrm{Su} \text { CA NaCl) }}$ to be used in subsequent data analyses. Data visualizations were created with GraphPad Prism (scatterplots) and ggplot2 package for RStudio.

fMRI analysis. Data were analyzed on Linux workstations using MATLAB R2011a (MathWorks) and SPM12 (Wellcome Trust Center for Neuroimaging, London, UK). All functional images from each participant were corrected for geometric distortions caused by susceptibility-induced field inhomogeneities. A combined approach was used that corrects for both static distortions and changes in these distortions attributable to head motion (Andersson et al., 2001; Hutton et al., 2002). The static distortions were calculated for each participant by acquiring a field map and processing it using the FieldMap toolbox implemented in SPM12 (Hutton et al., 2004). The images were then realigned, unwarped, and coregistered to the participant's own anatomic T1 image. The anatomic T1 image was processed using a unified segmentation procedure combining segmentation, bias correction, and spatial normalization (Ashburner and Friston, 2005); the same normalization parameters were then used to normalize the functional images. Then, all functional images were detrended using a method for removing any linear components matching the global signal at each voxel (Macey et al., 2004). Finally, functional images were smoothed with a $6 \mathrm{~mm}$ FWHM isotropic Gaussian kernel.

For the time-series analysis on all participants, a high-pass filter (300s) was included in the filtering matrix (adjusted from the convention in SPM12 to reflect the maximal period between two blocks of tasteless) to remove low-frequency noise and slow drifts in the signal. Conditionspecific effects at each voxel were estimated using the general linear model (Friston et al., 1995; Worsley and Friston, 1995). The response to events was modeled using a canonical hemodynamic response function included in SPM12. The temporal derivative of the hemodynamic response function was also included as part of the basis set to account for up to $1 \mathrm{~s}$ shifts in the timing of the events (Henson et al., 2002). There were two events of interest: "taste" and "tasteless." For taste, taste blocks taste $(\mathrm{Su})$, taste $(\mathrm{CA})$, and taste $(\mathrm{NaCl})$ were collapsed into a single event "taste( $\mu \mathrm{Su} \mathrm{CA} \mathrm{NaCl),"} \mathrm{referring} \mathrm{to} \mathrm{the} \mathrm{mean} \mathrm{response} \mathrm{to} \mathrm{these}$ tastants, parallel to the averaging of the ratings in the analysis for psychophysics. The corresponding tasteless blocks were also collapsed into a single event, "tasteless." Rinses, taste $(\mathrm{Qu})$, and tasteless $(\mathrm{Qu})$ blocks were each modeled as a nuisance effect. The Artifact Detection Tools (ART) toolbox (https://www.nitrc.org/projects/artifact_detect/) for MATLAB was used to detect global mean and motion outliers in the functional data. Motion parameters were included as regressors in the design matrix at the single-subject level. In addition, image volumes in which the $z$-normalized global brain activation exceeded 3 SDs from the mean of the run or showed $1 \mathrm{~mm}$ of composite (linear plus rotational) movement were flagged as outliers and deweighted during SPM estimation. We additionally calculated a composite movement estimate for each participant, frame-to-frame displacement. Frame-to-frame displacement was calculated by taking the Euclidean distance between the center of gravity of an image and the next image (Satterthwaite et al., 2013). We summed frame-to-frame displacement for all images within a run and subsequently averaged them across runs.

To assess group-level main effects of versus Tasteless independent of differences in individual sensitivity, the parameter estimate images for each participant were entered into a one-sample $t$ test using individual contrasts of taste $(\mu \mathrm{Su} \mathrm{CA} \mathrm{NaCl)-tasteless.} \mathrm{We} \mathrm{also} \mathrm{included} \mathrm{all} \mathrm{individ-}$ ual grand average scores of taste intensity ratings and composite movement estimates. To test the hypothesis that response in the amygdala would correlate with overall subjective taste intensity perception, we regressed individual grand average scores of taste intensity against BOLD response to taste $(\mu \mathrm{Su} \mathrm{CA} \mathrm{NaCl})$-tasteless across all 28 
participants using a small volume correction (sphere with $6 \mathrm{~mm}$ radius, containing 123 voxels) defined from a region of amygdala that we have previously shown codes taste intensity perception (at coordinate [(-) 24 $-6-21])$ [30]. The $t$ map threshold was set at $p_{\text {uncorrected }}<0.001$ and a cluster size at a minimum of 5 contiguous voxels. Unpredicted clusters were considered significant at $p<0.05$, false discovery rate corrected across the whole brain.

Psychophysiological interaction (PPI) analysis (Friston et al., 1997) was used to identify functional connectivity between the area of the amygdala sensitive to taste intensity perception and earlier gustatory relays (insular cortex, thalamus, and brainstem) using small volume corrections (sphere with $6 \mathrm{~mm}$ radius, containing 123 voxels) defined by centroids from two prior meta-analyses (at coordinates: [(-)8 $8-268$, and (-)10-14-8]; Veldhuizen et al., 2011a; Yeung et al., 2017). We used two meta-analyses because the first only reported thalamic responses in the mediodorsal (md) thalamus, and we wished to include the gustatory thalamus. Peaks were corrected for multiple comparison across the voxels within the small volumes and then corrected for the number of regions included (insula, thalamus, and brainstem). Regions that showed a main effect of taste $(\mu \mathrm{Su} \mathrm{CA} \mathrm{NaCl})$-tasteless at the group level or a relation with individual grand average scores were used as seed regions. The first eigenvariate of the time-series data were extracted from a $6 \mathrm{~mm}$ sphere with a centroid on the coordinate of the amygdala cluster. The eigenvariate was then deconvolved (Gitelman et al., 2003), multiplied with the psychological variable (taste $(\mu \mathrm{Su} \mathrm{CA} \mathrm{NaCl})$-tasteless), and reconvolved with a hemodynamic response function to form the PPI term. For each participant, we computed new parameter estimate images with the PPI as a regressor of interest and the time-series eigenvariate and psychological variable as nuisance regressors. In order to examine which areas display increased or decreased connectivity with the seed regions associated with intensity perception, we then regressed individual grand average scores of taste intensity ratings against PPI parameter estimate images across all 28 participants.

DCM was used to characterize associations between individual sensitivity to taste and neural circuitry in a network of regions responding to taste $(\mu \mathrm{Su} \mathrm{CA} \mathrm{NaCl})$-tasteless. We selected the seed and target regions that resulted from using the central amygdala PPI analysis. We specified a single fully connected and driven DCM between these regions. The DCM specified driving inputs (taste and tasteless regressors) into the network at all five regions. The DCM also specified a complete connectivity matrix for intrinsic, steadystate, bidirectional connections among the five regions. No modulatory effects on the connections between regions were specified, as they are not obligatory (Friston et al., 2003) and the design included no within-participant variables qualifying for modulation in the DCM. We first estimated the full DCM for each individual participant. We then used the second-level parametric empirical Bayes framework (Friston et al., 2016) pruning approach to reduce the model to a configuration of parameters to best explain the data observed in the regions. We used the pruning approach separately for the driving inputs and connectivity matrix (Zeidman et al., 2019a). We created a second-level general linear model with a design matrix that includes a constant factor, a regressor-of-interest for the individual grand average scores of taste intensity, and composite movement estimates as a nuisance variable. This results in a (Bayesian) hierarchical model, with estimates for connection strengths (positive or negative) between regions and their uncertainty (i.e., posterior probability; Zeidman et al., 2019b). There are two sets of estimates, as follows: (1) for a model that describes the average neural circuitry across participants, independent of individual sensitivity to taste; and (2) for a model that describes the neural circuitry associated with individual grand average scores of taste intensity. We display any parameters that showed a probability of $>0.90$. We used all connectivity parameters to predict individual grand average scores of taste intensity, using a leave-one-out crossvalidation (LOOCV) framework. In this regression procedure, a model is fitted to data from all but one participant and a prediction of the score is made for that participant, which is repeated in turn for all participants. This results in a Pearson's correlation between predicted and empirical intensity scores. We used an $\alpha$ of 0.05 to determine significance for this correlation.

Data visualizations were created with GraphPad Prism (GraphPad Software) for scatterplots, ggplot2 for boxplots (R-studio), Mango by Research Imaging Institute, UTHSCSA (for SPMs overlaid on brain sections of the BigBrain (Amunts et al., 2013), and Illustrator for DCM models (Adobe).

\section{Data availability}

Raw fMRI data are available upon request. Contact dana.small@yale. edu.

Statistical maps of the human brain are available on NeuroVault repository: https://identifiers.org/neurovault.collection:7635.

\section{Results}

\section{Taste psychophysics}

We first set out to determine whether prior findings of high cross-correlations between taste intensity ratings were replicated (Green and George, 2004; Lim et al., 2008). The four taste solutions were all rated as stronger in intensity than tasteless (Fig. 2; main effect of taste stimulus: $F_{(1,135)}=69.14, p<0.001$; sweettasteless: $t_{(27)}=9.83, p<0.001$; sour-tasteless: $t_{(27)}=8.94, p<$ 0.001 ; salty-tasteless: $r=t_{(27)}=9.94, p<0.001$; bitter-tasteless: $\left.t_{(27)}=8.51, p<0.001\right)$, while the taste solutions were not rated significantly different in intensity from each other (all corrected $p$ values $>0.826$ ). Pearson correlation analysis showed that intensity ratings of the four taste qualities positively correlated with each other (sweet-sour, $r=0.776$; sweet-salty, $r=0.855$; sweet-bitter, $r=0.373$; sour-salty, $r=0.807$; sour-bitter, $r=0.387$; and salty-bitter, $r=0.417$; Fig. 2); however, the correlation between quinine and the other taste solutions did not survive correction for multiple comparisons. The latter result is consistent with significant individual differences that have been found even among the sensitivities to different bitter compounds (Yokomukai et al., 1993; Delwiche et al., 2001), which has been proposed to result from differences in receptor expression among the 25 bitter taste receptors in humans (Meyerhof et al., 2010). Given these differences, a lower correlation between bitter and other tastes is not unexpected. A principal component analysis confirmed the existence of two components underlying the variance across participants, the first component accounted for $72 \%$ of the variance and received loadings from sweet, sour, and salty intensity, and the second component accounted for $19 \%$ of the variance and received a loading from quinine. Note also that the tasteless solution showed no significant correlation with any of the four prototypical tastes (sweet-tasteless, $r=0.061$; sourtasteless, $r=-0.109$; salty-tasteless, $r=0.119$; bitter-tasteless, $r=0.041$ ). Thus, we calculated grand intensity scores for each participant's taste intensity ratings by averaging ratings for sweet, sour, and salty tastants, referred to hereafter as the mean log intensity $^{\mu(\mathrm{Su} \mathrm{CA} \mathrm{NaCl})}$. The Pearson correlation matrix between grand intensity scores and the intensity scores of the four taste solutions are shown in Figure 2, including all $r$ values (effect size) and $p$ values (corrected for multiple comparisons).

\section{fMRI data}

Main effect of taste versus tasteless

Consistent with previous studies, a main effect of taste( $\mu$ Su NA $\mathrm{NaCl}$ )-tasteless was observed in bilateral md and anterior-ventral insula, and extending into overlying operculum, bilateral preand post-central gyrus, left amygdala, dorsal anterior cingulate cortex, and left putamen (Fig. 3, Table 1). 


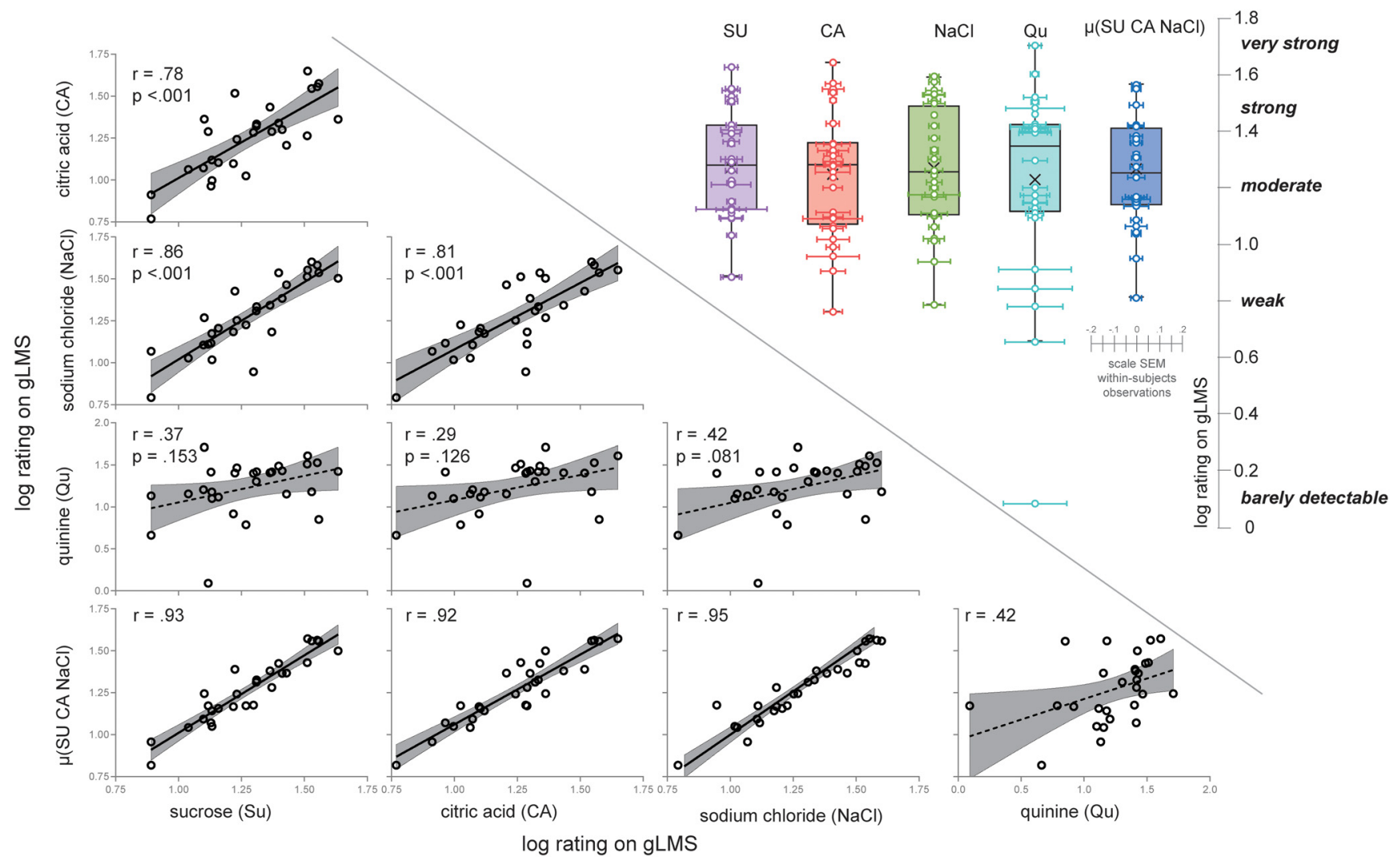

Figure 2. Taste intensity ratings. Below the diagonal: scatterplots of log-transformed intensity ratings on the gLMS for all tastant pairs and each tastant with the average of the three significantly correlated tastants (from top to bottom: citric acid, sodium chloride, quinine, and mean $\log$ intensity ${ }^{\mu(\text { Su }}$ CA NaCl); from left to right sucrose, citric acid, sodium chloride, quinine). Each datapoint represents one participant. Above the diagonal, The boxplots indicate central tendencies and spread of the log-transformed intensity rating ( $y$-axis on far right), as follows: average (represented by a cross symbol), median (middle bar in box), first and third quartiles (lower and upper hinge), $1.5 \times$ the interquartile range (top and bottom whiskers) and outlying points (separate dots outside the whiskers). We overlaid individual data points (one for each participant) on the boxplots with within-participant SEM of the average of eight ratings they gave for each tastant represented as horizontal error bars. From left to right, the boxplots represent the following: sucrose, citric acid, sodium chloride, quinine, and mean log intensity ${ }^{\mu(S u}$ (A Nacl). For interpretation, gLMS label placement is given on the $y$-axis on the far right.

Regression of BOLD response with mean log intensity ${ }^{\mu(\mathrm{Su} \mathrm{CA} \mathrm{NaCl})}$ To identify regions sensitive to overall gustatory system sensitivity, we used the mean $\log$ intensity ${ }^{\mu(\mathrm{Su} \mathrm{CA} \mathrm{NaCl})}$ as the independent variable in a regression analysis with the BOLD response to taste $(\mu \mathrm{Su} \mathrm{CA} \mathrm{NaCl})$-tasteless. As predicted, the BOLD response in the left central amygdala was significantly associated with mean $\log$ intensity $^{\mu(\mathrm{Su} \mathrm{CA} \mathrm{NaCl})}$. We also observed significant associations with response in the cuneus (Fig. 4, Table 2). Notably, response in insular cortex did not correlate with the mean intensity rating. We also note that neither swallowing estimates $\left(r_{(22)}=-0.051, p=0.819\right.$; see Extended Data Materials) nor composite movement estimates $\left(r_{(27)}=-0.124, p=0.531\right)$ correlate with the mean intensity ratings.

\section{Functional connectivity of the CGM}

Next, to identify regions of the brain where functional connectivity with the amygdala is influenced by taste sensitivity, we first performed a PPI analysis (Friston et al., 1997) and then regressed the responses of areas displaying significant functional connectivity with the mean $\log$ intensity $\mu(\mathrm{Su} \mathrm{CA} \mathrm{NaCl})$. The presence of functional connectivity indicates that the connected regions display temporal correlation beyond that accounted for by the task and suggests that the regions form a functional network. The subsequent regression then determines which of these network associations are influenced by taste sensitivity. This analysis revealed amygdala functional connectivity with the md and ventral posterior medial/pulvinar (vpm/pul) thalamus (Fig. $5 A$, Table 3). We observed unpredicted functional connectivity in the mid-temporal gyrus (Table 3 ). No other significant effects were observed.

\section{Effective connectivity of the CGM}

Whereas PPI identifies correlations between regions, DCM can be used to establish effective connectivity, enabling inferences to be made about the influence that one neuronal system exerts over another (Friston et al., 2011). Based on the PPI and our hypothesized CGM, we predicted the existence of inhibitory modulatory influences from the amygdala to the thalamic nuclei, where the strength of the connection is associated with taste sensitivity. We therefore specified a DCM with five regions (left amygdala, bilateral $\mathrm{md} /$ thalamus, and bilateral $\mathrm{vpm} / \mathrm{pul}$ ) to examine the ensemble of connectivity parameters in relation to $\log$ intensity $^{\mu(\mathrm{Su} \mathrm{CA} \mathrm{NaCl})}$ and subjected this to Bayesian model estimation. The architecture of the selected model conformed to our prediction, as follows: inhibitory connections were identified from the amygdala to the four thalamic peaks (Fig. $5 B$ ). We also identified a positive bidirectional connection between right $\mathrm{md}$ and right vpm/pul. When we entered the connectivity parameters into a multivariate LOOCV regression, we found a significant association between predicted and observed mean $\log$ intensity $^{\mu(\mathrm{Su} \mathrm{CA} \mathrm{NaCl}}\left(r_{(27)}=0.37, p=0.027\right.$; Fig. $\left.5 B\right)$. To determine which of these connections contributed most to this predictive model, we used a univariate LOOCV approach, which 

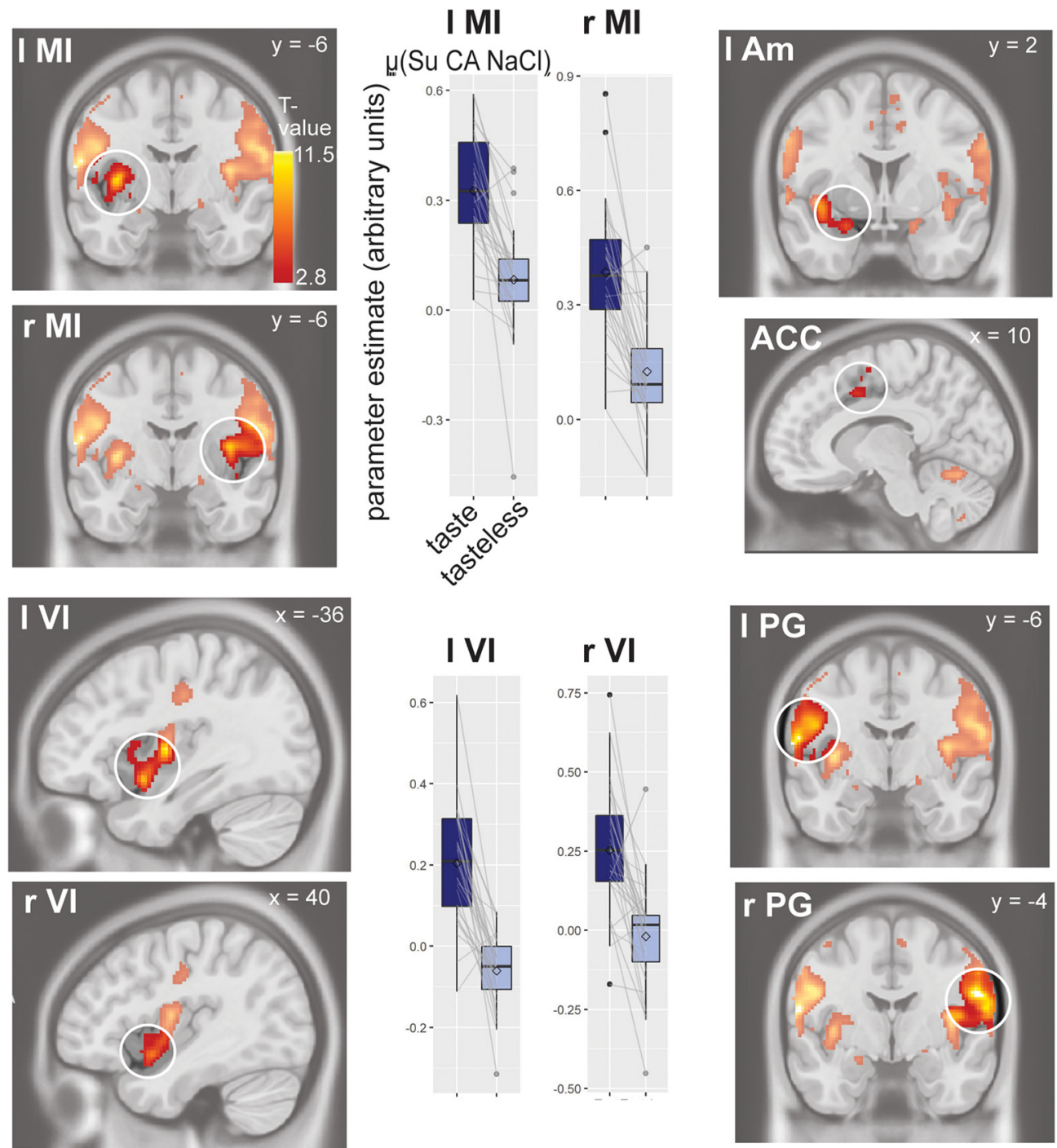

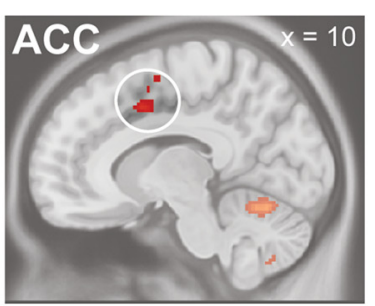

I Am

ACC
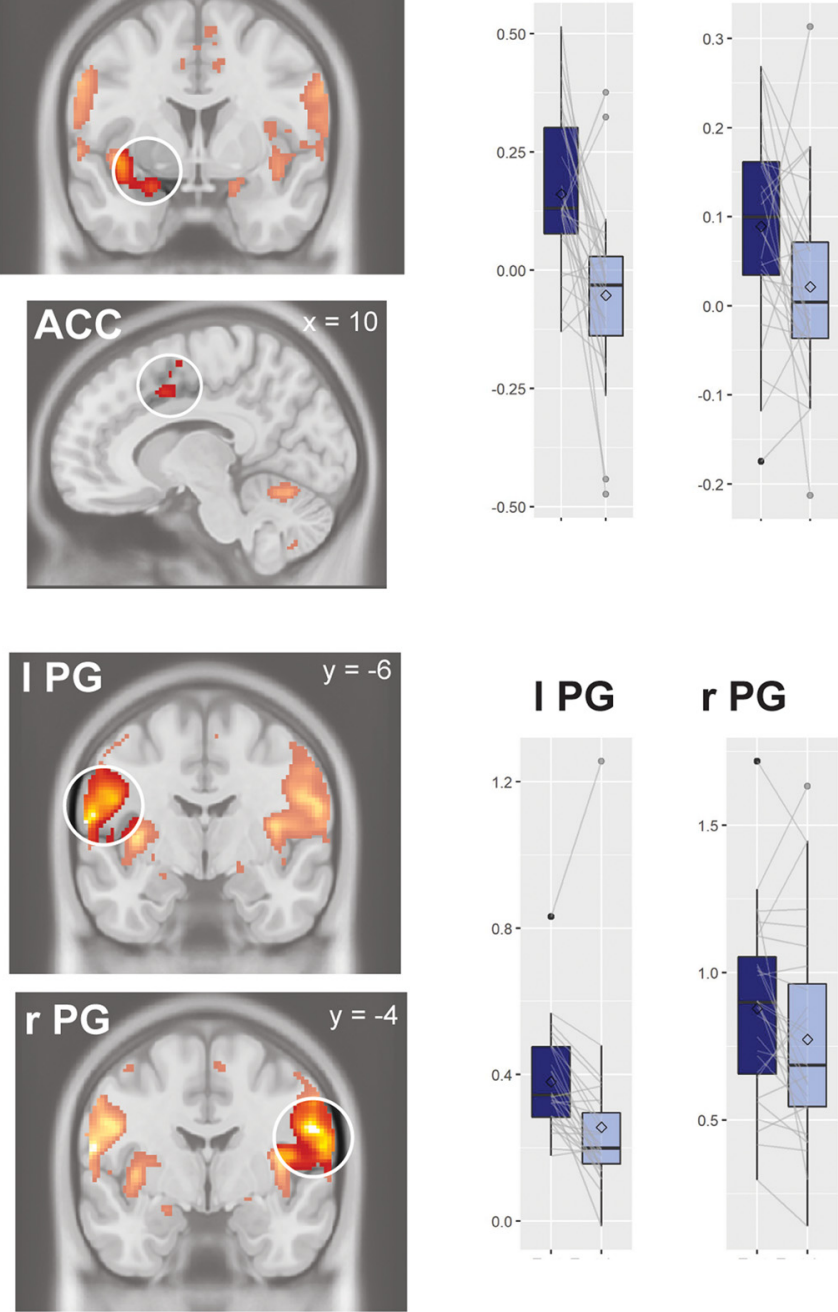

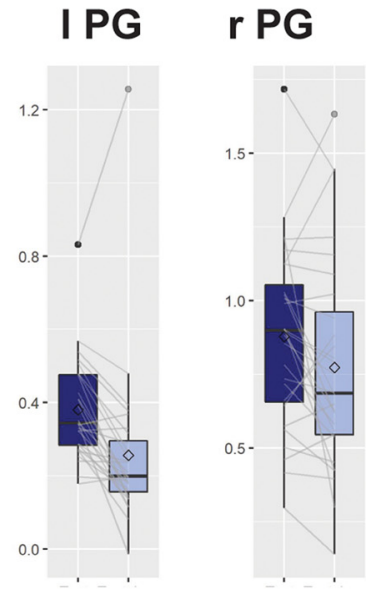

Figure 3. Neural response to taste( $\mu$ Su CA NaCl)-tasteless in insula, pre- and post-central gyrus, amygdala, and anterior cingulate cortex (ACC; key: r, right; I, left; MI, middle insula; VI, ventral insula; PG, pre- and post-central gyrus; AM, amygdala; ACC, anterior cingulate cortex). Sections (slice location indicated in MNI coordinates) show canonical anatomic template with SPM T-map overlaid, thresholded at $p_{\text {uncorrected }}<0.005$, and a minimum of five contiguous voxels. The color gradient scale depicts suprathreshold $t$ values. Boxplot graphs show median (center line), mean (diamond), first and third quartiles (lower and upper hinge), $1.5 \times$ the interquartile range (top and bottom whiskers), and outlying points (separate dots outside the whiskers). We plotted parameter estimate of the peak voxels within a significant cluster for the average of taste( $\mu$ Su CA NaCl)-tasteless. We overlaid individual data points on the boxplots and connected the dots of an individual participant between the taste and tasteless bars to make it easier to inspect the difference within a single participant.

revealed that the connections from amygdala to left vpm/pul and right md survived, as well as the positive connection from right $\mathrm{vpm} /$ pul to right $\mathrm{md}$.

\section{Discussion}

Significant individual differences exist in taste intensity perception. Some of these differences can be explained by peripheral mechanisms, such as genetic variation in taste receptor expression and biology. However, as noted in the Introduction, while peripheral mechanisms can play a significant role in individual differences in the perceived intensity of individual taste qualities or even specific tastants (e.g., PROP), they are not able to account for overall differences in gustatory system sensitivity, such as the high within-subject cross-correlations among intensity ratings of different taste qualities (Green and George, 2004; Green et al., 2005; Lim et al., 2008). These observations are suggestive of a central mechanism that affects the responsiveness to all taste stimulation. Here we used fMRI and taste psychophysics to identify a region of amygdala where the response to a series of tastants is correlated with overall gustatory system sensitivity and drives inhibitory outputs to the gustatory and limbic nuclei of the thalamus. We further show that the dynamics and strength of these inhibitory connections are able to predict individual variation in taste intensity ratings.

Our primary hypothesis for a CGM focused on the amygdala for several reasons. First, patients with amygdala removal for the treatment of epilepsy show enhanced taste, but not visual, intensity ratings (Small et al., 1997, 2001a,b). This enhanced perception suggests the existence of an inhibitory influence from the amygdala to the gustatory network, which when removed releases the target regions from inhibition to enhance the taste signal. Second, our prediction aligns with the known anatomy and physiology of the amygdala. The amygdala can be broadly subdivided into the basolateral 
Table 1. Significant clusters of BOLD response to taste( $\mu$ Su CA NaCl)-tasteless

\begin{tabular}{|c|c|c|c|c|c|c|}
\hline \multirow[b]{2}{*}{ Label (BA) } & \multirow[b]{2}{*}{$p_{\text {FWE }}$} & \multirow{2}{*}{$\begin{array}{l}\text { Cluster } \\
\text { size }\end{array}$} & \multirow[b]{2}{*}{$t$ Value } & \multicolumn{3}{|l|}{$\mathrm{MNI}$} \\
\hline & & & & $x$ & $y$ & $z$ \\
\hline Postcentral gyrus (BA 43) & $<0.001^{*}$ & 3119 & 11.485 & -64 & -6 & 14 \\
\hline Middle insula (posterior short gyrus) & & & 11.238 & -36 & -8 & 6 \\
\hline Precentral gyrus (BA 6) & & & 9.468 & -50 & -6 & 26 \\
\hline Ventral insula (anterior inferior cortex) & & & 8.951 & -36 & 6 & -12 \\
\hline Precentral gyrus (BA 4) & & & 8.581 & -44 & -14 & 36 \\
\hline Precentral gyrus (BA 6) & & & 8.282 & -58 & 0 & 36 \\
\hline Ventral insula (anterior inferior cortex) & & & 8.145 & -40 & 2 & -6 \\
\hline Postcentral gyrus (BA 1) & & & 7.669 & -64 & -14 & 24 \\
\hline Superior temporal gyrus (BA 22) & & & 5.875 & -60 & 2 & 0 \\
\hline Amygdala/anterior piriform & & & 5.429 & -24 & 2 & -18 \\
\hline Superior temporal gyrus (BA 22) & & & 5.134 & -56 & 12 & -6 \\
\hline Lentiform nucleus putamen & & & 5.059 & -28 & -10 & -4 \\
\hline Precentral gyrus (BA 4) & & & 4.287 & -46 & -10 & 58 \\
\hline Inferior frontal gyrus (BA 45) & & & 4.270 & -60 & 12 & 18 \\
\hline Posterior insula (anterior short gyrus) & & & 3.825 & -40 & -14 & -8 \\
\hline Anterior insula (middle short gyrus) & & & 3.523 & -34 & 10 & 8 \\
\hline Precentral gyrus (BA 6) & $<0.001^{*}$ & 2572 & 9.906 & 54 & -4 & 28 \\
\hline Middle insula (posterior short gyrus) & & & 9.449 & 38 & -6 & 12 \\
\hline Frontal operculum & & & 8.036 & 48 & -8 & 12 \\
\hline Postcentral gyrus (BA 43) & & & 7.552 & 66 & -12 & 22 \\
\hline Precentral gyrus (BA 6) & & & 7.227 & 62 & 2 & 32 \\
\hline Ventral insula (anterior inferior cortex) & & & 6.902 & 40 & 6 & -12 \\
\hline Precentral gyrus (BA 6) & & & 6.374 & 56 & -6 & 42 \\
\hline Middle insula (anterior long gyrus) & & & 5.674 & 38 & -2 & -2 \\
\hline Superior temporal gyrus (BA 22) & & & 5.137 & 58 & 12 & -2 \\
\hline Precentral gyrus (BA 44) & & & 5.083 & 64 & 8 & 12 \\
\hline Superior temporal gyrus (BA 22) & & & 4.555 & 64 & -2 & 4 \\
\hline Anterior insula (posterior short gyrus) & & & 4.391 & 36 & 8 & 10 \\
\hline Precentral gyrus (BA 40 & & & 3.986 & 38 & -16 & 40 \\
\hline Ventral insula (anterior inferior cortex) & & & 3.712 & 40 & 8 & -2 \\
\hline Precentral gyrus (BA 6) & & & 3.469 & 48 & -6 & 54 \\
\hline Frontal operculum & & & 3.272 & 46 & 4 & 10 \\
\hline Frontal operculum & & & 3.271 & 52 & 4 & 4 \\
\hline Cerebellum posterior lobe declive & $<0.001^{*}$ & 805 & 9.182 & 14 & -60 & -18 \\
\hline Cerebellum posterior lobe declive & & & 7.376 & -16 & -62 & -20 \\
\hline Cerebellum posterior lobe declive & & & 6.403 & 4 & -68 & -16 \\
\hline Cerebellum posterior lobe declive & & & 3.353 & -30 & -62 & -18 \\
\hline
\end{tabular}

$\mathrm{BA}$, Brodmann area.

${ }^{*} p$ value for $\alpha=0.05$ FWE-corrected for multiple comparisons at the cluster-level across the whole brain. Bold font indicates peak voxel.

complex, which receives sensory inputs, and a central medial division that produces the primary efferent information. Central nucleus efferent projections tend to be inhibitory and play an important role in regulating innate emotional responses and their associated autonomic outflow via connections to brainstem nuclei and md thalamus (Aggleton and Mishkin, 1984; Swanson and Petrovich, 1998; Barbas, 2000; Fadok et al., 2018). Accordingly, and in line with our prediction, we observed a correlation between the response to taste in the central medial nucleus of the amygdala and the mean log intensity ratings to sweet, sour, and salty tastants. Further, our connectivity analyses revealed inhibitory influences from this region to the md and gustatory (i.e., VPM) thalamic nuclei.

In primates, including humans, taste information ascends from the periphery to the rostral NST (Beckstead and Norgren, 1979). In rodents, after an obligatory synapse in the parabrachial pons, the gustatory pathway bifurcates at this point with one projection targeting the amygdala and hypothalamus and a second projection targeting the VPM gustatory thalamus, which subsequently projects to insular taste cortex (Norgren and Leonard, 1971; Norgren, 1976, 1984, 1990; Kosar et al., 1986). In primates, there is no bifurcation and only a single projection from NST to VPM and finally insular taste cortex (Beckstead et al., 1980; Pritchard et al., 1986, 2000). Given this significant interspecies difference, we formed our prediction from the anatomic work in nonhuman primates and reasoned that the target for the inhibitory signal from the amygdala could be NST, thalamus, or insula (and not pons). However, the results from the PPI, which evaluated functional connectivity between the amygdala and the rest of the voxels in the brain, identified selective bilateral connectivity with two thalamic nuclei, highlighting these nuclei as the primary targets for inhibitory modulation.

The md thalamus is not part of the canonical taste pathway. However, it does receive gustatory and olfactory input (Price and Slotnick, 1983; Shi and Cassell, 1998; Cavada et al., 2000), and, although amygdala-thalamic connectivity is understudied, there is some evidence for a direct projection from the central nucleus of the amygdala to the md thalamus (Aggleton and Mishkin, 1984). By contrast, reciprocal connections do not appear to exist (Aggleton et al., 1980), which is consistent with the lack of reciprocal connections revealed here in the DCM analysis. While activation of the md thalamus is frequently found in gustatory fMRI studies, little is understood about its role in gustation. Work in nonhuman primates shows that it projects to the posterior orbitofrontal cortex, which is a site of convergence for taste and smell information (De Araujo et al., 2003; Small et al., 2004) and is proposed to form a tripartite circuit to convey information about emotional (and presumably physiological) significance to afferent sensory information (Timbie and Barbas, 2015). Accordingly, in rodents c-Fos expression is greater for familiar tastes and taste odor mixtures, suggesting sensitivity to flavor nutrient conditioning and therefore physiological significance (Fredericksen et al., 2019).

The second thalamic activation was further posterior and closely corresponded to the thalamic region previously associated with taste intensity perception (Small et al., 2003; Yeung et al., 2016). However, the activation extended into pulvinar, which is of relevance because it is often coactivated with amygdala in response to salient but nongustatory stimuli (Morris et al., 1997, 1999). Importantly, the entire human thalamus is only $\sim 30 \times 20 \times 20 \mathrm{~mm}$, with the VPM occupying less than one-tenth of this volume (Herrero et al., 2002). Given our $2 \mathrm{~mm}^{3}$ voxel size and $6 \mathrm{~mm}$ smoothing kernel, the taste thalamus, which is the parvicellular division of the VPM, is beyond the limits of our spatial resolution. It is therefore likely that the cluster of response we have observed overlaps the taste nucleus. The VPM is the target of gustatory afferents and projects to the insular taste cortex. However, no known direct connections exist between central medial amygdala and VPM (Kaskan et al., 2019). How the inhibitory influences from the central medial amygdala reach the VPM thalamus to regulate perception is therefore unknown and an important topic for future work. A related issue is whether this circuit operates independently from the known basolateral amygdala influence on taste cortex, which has been shown to enhance detection of expected taste stimuli in rodents (Samuelsen et al., 2012). Here we observed no associations between overall intensity perception and basolateral amygdala response or connectivity. Another consideration is the well known role the amygdala plays in orienting to salient stimuli (Cunningham and Brosch, 2012), even when presentation is implicit (Morris et al., 1999). Since the primary function of taste sensation is to guide feeding decisions, 
A

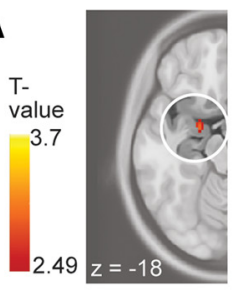

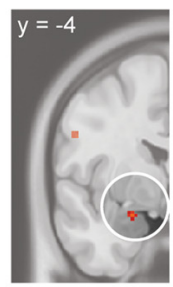
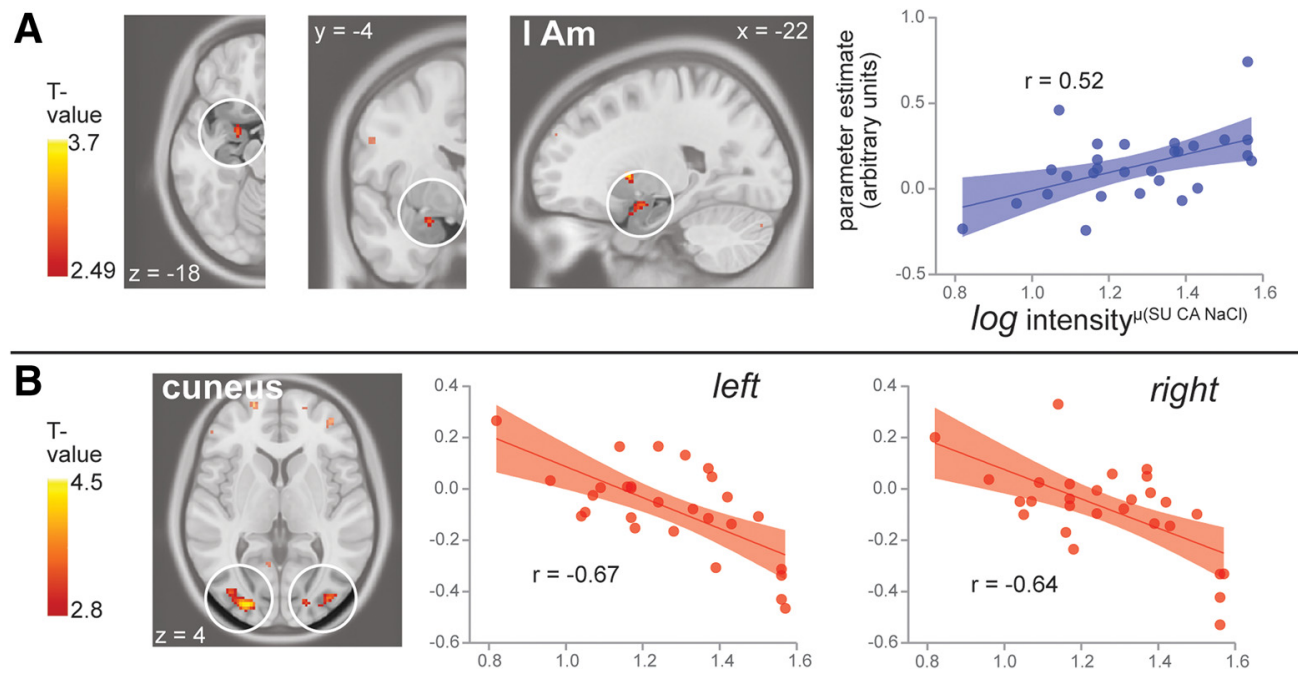

Figure 4. Neural response to taste( $\mu$ Su CA NaCl)-tasteless correlated with mean $\log$ intensity ${ }^{\mu \text { (Su CA NaCl) }}$ in $\boldsymbol{A}$, left amygdala (AM), and $\boldsymbol{B}$, bilateral cuneus. Brain slices are as described in Figure 3. Scatterplots show each participant's values, with mean $\log$ intensity ${ }^{\mu(S u}$ CA NaCl) on the $x$-axis and parameter estimate on the $y$-axis for the peak voxel within a significant cluster. Regression line with regression coefficient and $95 \%$ confidence interval bands are overlaid for illustrative purposes. Positive associations are shown in blue, and negative associations are shown in red.

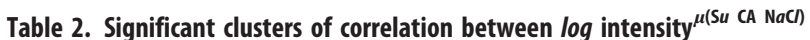
and BOLD response to taste( $\mu$ Su CA NaCl)-tasteless

\begin{tabular}{|c|c|c|c|c|c|c|}
\hline \multirow[b]{2}{*}{ Label (BA) } & \multirow[b]{2}{*}{$P_{\text {FWE }}$} & \multirow[b]{2}{*}{ Cluster size } & \multirow[b]{2}{*}{$t$ value } & \multicolumn{3}{|l|}{ MNI } \\
\hline & & & & $x$ & $y$ & $z$ \\
\hline Amygdala & $0.049^{\S}$ & 12 & 3.211 & -22 & -4 & -16 \\
\hline \multirow[t]{5}{*}{ Inferior occipital gyrus (BA 18) } & $0.007^{*}$ & 393 & 4.529 & 36 & -86 & -2 \\
\hline & & & 4.308 & 28 & -84 & 18 \\
\hline & & & 3.837 & 34 & -80 & 12 \\
\hline & & & 3.541 & 18 & -86 & 6 \\
\hline & & & 3.013 & 22 & -94 & 14 \\
\hline \multirow[t]{10}{*}{ Mid occipital gyrus (BA 18) } & $<0.001^{*}$ & 543 & 4.425 & -26 & -90 & 4 \\
\hline & & & 4.072 & -26 & -78 & 12 \\
\hline & & & 4.033 & -38 & -78 & 6 \\
\hline & & & 3.716 & -20 & -86 & -10 \\
\hline & & & 3.549 & -16 & -94 & 8 \\
\hline & & & 3.484 & -24 & -88 & 20 \\
\hline & & & 3.280 & -22 & -78 & 20 \\
\hline & & & 3.279 & -30 & -86 & -12 \\
\hline & & & 3.193 & -18 & -92 & 24 \\
\hline & & & 3.118 & -10 & -94 & 14 \\
\hline
\end{tabular}

BA, Brodmann area.

${ }^{\S} p$ value for $\alpha=0.05$ FWE-corrected for multiple comparisons at the voxel level across a small volume search (SVC). ${ }^{*} p$ value for $\alpha=0.05$ FWE-corrected for multiple comparisons at the cluster-level across the whole brain. Bold font indicates peak voxel.

amygdala "gating" of taste information could be part of a larger system devoted to the identification of salient stimuli and attentional allocation.

However, whereas amygdala lesions impair taste recognition (Small et al., 1997) similarly to the effects observed with other salient stimuli, such as fearful faces (Adolphs et al., 1994; Calder, 1996), opposing effects are seen with sensitivity. Specifically, sensitivity to taste is enhanced while sensitivity to visual and auditory stimuli that should evoke fear is severely blunted (Adolphs et al., 1994; Calder, 1996). Interestingly, taste identification is likely encoded in parallel with taste detection (Wallroth and Ohla, 2018), with evidence that taste intensity is computed at the perceptual (Veldhuizen et al., 2010) and neural (Katz et al., 2002) levels before identity and hedonic significance. These observations suggest that the CGM may operate on the earliest stages of gustatory processing. Accordingly, the CGM may function to modulate taste and olfactory stimulation to increase the saliency of significant flavor stimuli in the service of selective attention or in the course of associative learning. Indeed, CGMs have been proposed and studied as a mechanism of selective attention in vision and hearing (Connor et al., 1996; Hillyard et al., 1998; Kauramäki et al., 2007; Womelsdorf and Fries, 2007; Jacobs et al., 2012). In this view, individual differences in the perceived intensity of tastes and odors would be attributable in part to differences in the baseline "gain" of such a system. The alternative explanation is that the amygdala response reflects a downstream effect of an earlier modulatory mechanism. However, while the correlational nature of fMRI data means that it is not possible to rule this possibility out, it is unlikely to account for our findings. First, a peripheral mechanism cannot account for the across-tastant and across-modal (olfactory) nature of the effect (Green and George, 2004; Green et al., 2005) and, second, neither can the insular cortex response, which reflects the first cortical relay, because it was not correlated with mean intensity ratings.

Relatedly, there is evidence for opposing responses in gustatory and visual streams of processing, especially regarding allocation of attentional resources. For example, breaches of taste expectation that occur when an individual expects to sense one tastant (e.g., sweet) but receives another (e.g., tasteless) are associated with enhanced response in gustatory, attentional, and error signaling regions coupled with decreased response in visual cortex (Veldhuizen et al., 2011b). Likewise, here we observed positive functional connectivity between the amygdala and the thalamic targets and negative functional connectivity between the amygdala and visual cortex. This suggests that the amygdala may play a role in coordinating the allocation of resources to visual (or distal) versus gustatory (or proximal/visceral) sensory systems, depending on the behavioral context or state of the organism.

We scanned with a multiband sequence to increase spatial coverage and spatial and temporal resolution. Slice leakage artifacts may result from multiband sequences and lead to false positive results (Todd et al., 2016). Here we used a combination of 

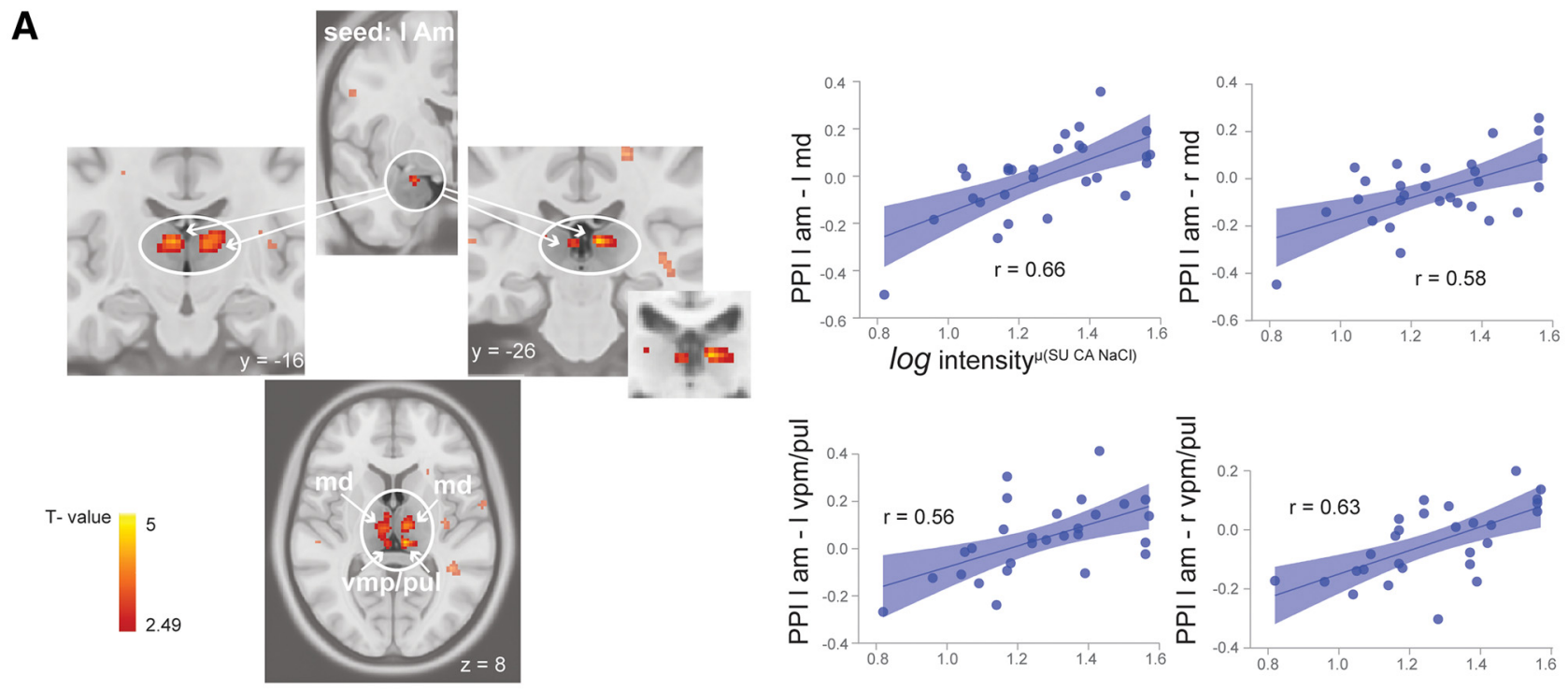

B
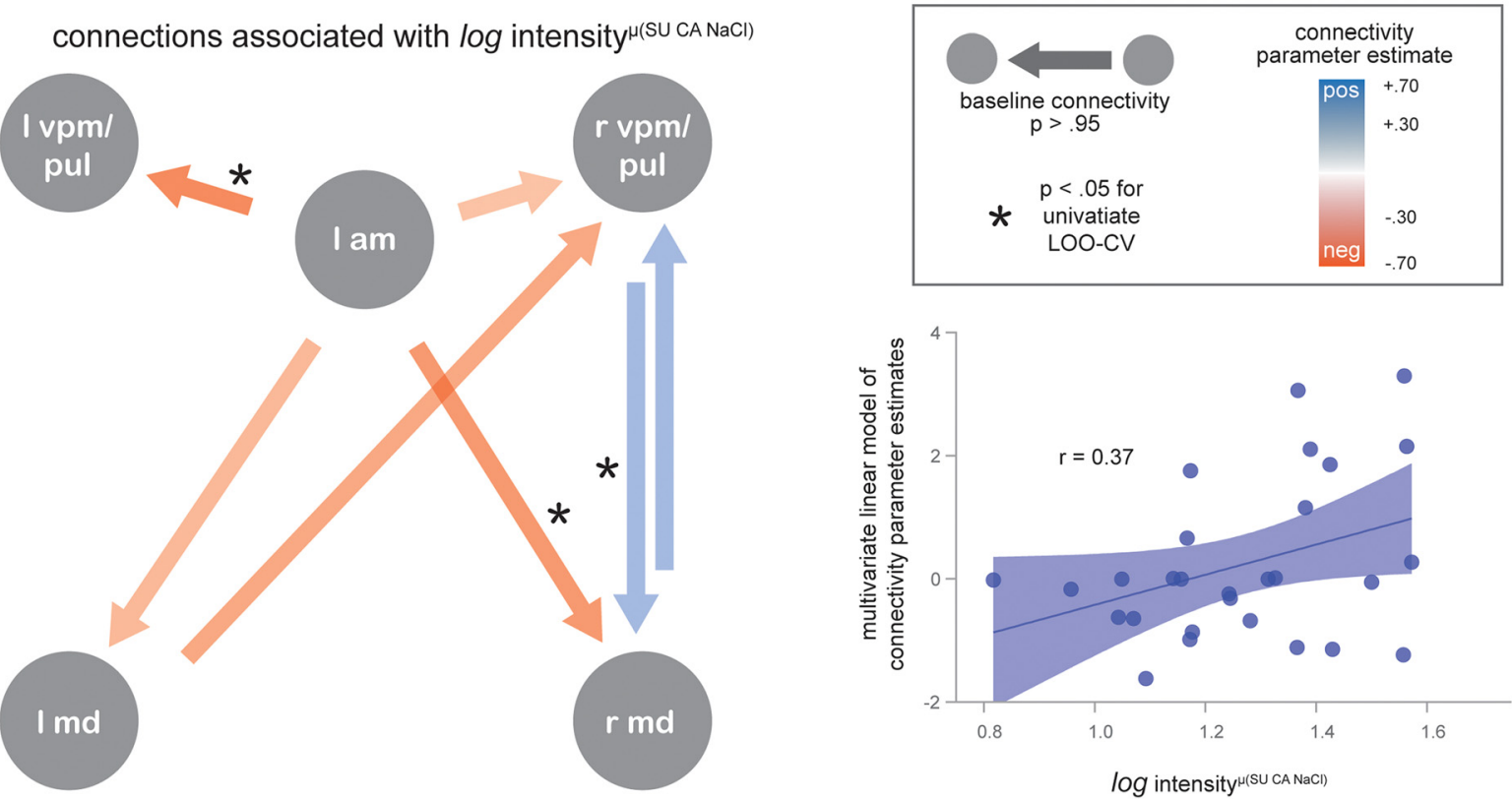

Figure 5. Connectivity correlated with mean $\log$ intensity ${ }^{\mu(\mathrm{Su} \text { CA NaCl) }} \cdot \boldsymbol{A}$, Connectivity parameter estimates from PPI analysis between seed region in left amygdala (am) and regions in thala-

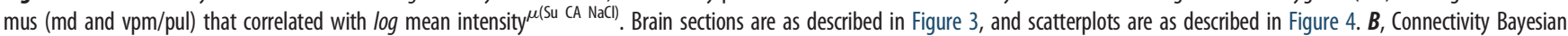
estimates in the DCM of the amygdala and thalamus areas. The gradient of arrows indicates the estimates for connection strengths (positive in orange or negative in blue) between regions for any connections with a posterior probability of $p>0.95$. The scatterplot shows each participant's values from the multivariate LOOCV regression, with mean $\log$ intensity ${ }^{\mu(S u}$ CA NaCl) on the $x$ axis and connectivity parameter estimate of the multivariate linear DCM model on the $y$-axis. The regression line with regression coefficient and $95 \%$ confidence interval bands is overlaid for illustrative purposes. Asterisks in the model indicate the connection that survived univariate LOOCV regression.

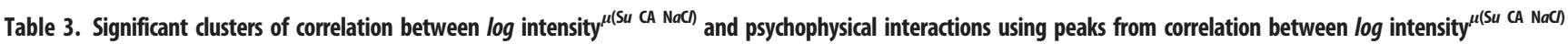
(see Table 2)

\begin{tabular}{|c|c|c|c|c|c|c|c|}
\hline \multirow[b]{2}{*}{ Seed region } & \multirow[b]{2}{*}{ Label } & \multirow[b]{2}{*}{$p_{\text {FWE }}$} & \multirow[b]{2}{*}{ Cluster size } & \multirow[b]{2}{*}{$t$ Value } & \multicolumn{3}{|l|}{ MNI } \\
\hline & & & & & $x$ & $y$ & $z$ \\
\hline \multirow[t]{3}{*}{ Amygdala/anterior piriform } & Mid-temporal gyrus & 0.009 & 279 & 4.94 & -48 & -38 & -4 \\
\hline & & & & 3.40 & -48 & -20 & -14 \\
\hline & & & & 3.06 & -64 & -24 & -12 \\
\hline \multirow[t]{2}{*}{ Amygdala/anterior piriform } & Ventral posterior medial thalamus/medial pulvinar & $0.012^{\S}$ & 37 & 4.66 & 8 & -26 & 8 \\
\hline & Medio-dorsal thalamus & $0.027^{\S}$ & 70 & 4.23 & 10 & -14 & 12 \\
\hline
\end{tabular}

${ }^{5} p$ value for $\alpha=0.05$ FWE-corrected for multiple comparisons at the voxel level across a small volume search (SVC), subsequently $p$ value Bonferroni corrected for multiple SVC searches. Bold font indicates peak voxel. 
acceleration parameters that are at an intermediate level of signal-to-noise ratio and have an intermediate probability of obtaining a false-positive result. This was aimed to strike a good balance between higher signal-to-noise ratio and avoiding false positive results.

Finally, we note that although we excluded bitter taste from our analyses, we do not suggest that bitter tastants are unaffected by the CGM. Rather, it is likely that central factors are harder to detect because of the significant genetic variation in bitter taste receptors (Kim et al., 2004; Drayna, 2005), causing bitter taste perception to be more strongly influenced by peripheral mechanisms (Meyerhof et al., 2010; Hayes et al., 2015).

Here we identify a region of amygdala where the response to a series of tastants is correlated with overall gustatory system responsiveness and drives inhibitory outputs to the gustatory and limbic nuclei of the thalamus. Since the dynamics and strength of these inhibitory connections are able to predict individual variation in taste intensity ratings, we suggest that this circuit acts to create gain in the gustatory system to regulate taste intensity perception, and that the magnitude of the gain contributes to individual differences in taste perception.

\section{References}

Adolphs R, Tranel D, Damasio H, Damasio A (1994) Impaired recognition of emotion in facial expressions following bilateral damage to the human amygdala. Nature 372:669-672.

Aggleton JP, Burton MJ, Passingham RE (1980) Cortical and subcortical afferents to the amygdala of the rhesus monkey (Macaca mulatta). Brain Res 190:347-368.

Aggleton JP, Mishkin M (1984) Projections of the amygdala to the thalamus in the cynomolgus monkey. J Comp Neurol 222:56-68.

Allen AL, McGeary JE, Hayes JE (2014) Polymorphisms in TRPV1 and TAS2Rs associate with sensations from sampled ethanol. Alcohol Clin Exp Res 38:2550-2560.

Amunts K, Lepage C, Borgeat L, Mohlberg H, Dickscheid T, Rousseau M-É, Bludau S, Bazin P-L, Lewis LB, Oros-Peusquens A-M, Shah NJ, Lippert T, Zilles K, Evans AC (2013) BigBrain: an ultrahigh-resolution 3D human brain model. Science 340:1472-1475.

Andersson JLR, Hutton C, Ashburner J, Turner R, Friston K (2001) Modeling geometric deformations in EPI time series. Neuroimage 13:903-919.

Ashburner J, Friston KJ (2005) Unified segmentation. Neuroimage 26:839851.

Barbas H (2000) Connections underlying the synthesis of cognition, memory, and emotion in primate prefrontal cortices. Brain Res Bull 52:319-330.

Bartoshuk LM, McBurney DH, Pfaffmann C (1964) Taste of sodium chloride solutions after adaptation to sodium chloride: implications for the "water taste". Science 143:967-968.

Bartoshuk LM, Duffy VB, Green BG, Hoffman HJ, Ko C-W, Lucchina LA, Marks LE, Snyder DJ, Weiffenbach JM (2004) Valid across-group comparisons with labeled scales: the gLMS vs magnitude matching. Physiol Behav 82:109-114.

Beckstead RM, Norgren R (1979) An autoradiographic examination of the central distribution of the trigeminal, facial, glossopharyngeal, and vagal nerves in the monkey. J Comp Neurol 184:455-472.

Beckstead RM, Morse JR, Norgren R (1980) The nucleus of the solitary tract in the monkey: projections to the thalamus and brain stem nuclei. J Comp Neurol 190:259-282.

Breslin PAS (2013) An evolutionary perspective on food and human taste. Curr Biol 23:R409-R418.

Calder AJ (1996) Facial emotion recognition after bilateral amygdala damage: differentially severe impairment of fear. Cogn Neuropsychol 13:699-745.

Cavada C, Compañy T, Tejedor J, Cruz-Rizzolo RJ, Reinoso-Suárez F (2000) The anatomical connections of the macaque monkey orbitofrontal cortex. A review. Cereb Cortex 10:220-242.
Connor CE, Gallant JL, Preddie DC, Van Essen DC (1996) Responses in area V4 depend on the spatial relationship between stimulus and attention. J Neurophysiol 75:1306-1308.

Cruz A, Green BG (2000) Thermal stimulation of taste. Nature 403:889-892.

Cunningham WA, Brosch T (2012) Motivational salience: amygdala tuning from traits, needs, values, and goals. Curr Dir Psychol Sci 21:54-59.

Daunizeau J, Preuschoff K, Friston K, Stephan K (2011) Optimizing experimental design for comparing models of brain function. PLoS Comput Biol 7:e1002280.

De Araujo IE, Rolls ET, Kringelbach ML, McGlone F, Phillips N (2003) Taste-olfactory convergence, and the representation of the pleasantness of flavour, in the human brain. Eur J Neurosci 18:2059-2068.

Delwiche JF, Buletic Z, Breslin PAS (2001) Covariation in individuals' sensitivities to bitter compounds: evidence supporting multiple receptor/ transduction mechanisms. Percept Psychophys 63:761-776.

Drayna D (2005) Human taste genetics. Annu Rev Genomics Hum Genet 6:217-235.

Duffy VB, Bartoshuk LM (2000) Food acceptance and genetic variation in taste. J Am Diet Assoc 100:647-655.

Fadok JP, Markovic M, Tovote P, Lüthi A (2018) New perspectives on central amygdala function. Curr Opin Neurobiol 49:141-147.

Feeney EL, Hayes JE (2014) Regional differences in suprathreshold intensity for bitter and umami stimuli. Chemosens Percept 7:147-157.

Fredericksen KE, McQueen KA, Samuelsen CL (2019) Experience-dependent c-Fos expression in the mediodorsal thalamus varies with chemosensory modality. Chem Senses 44:41-49.

Frey S, Petrides M (1999) Re-examination of the human taste region: a positron emission tomography study: positron emission tomography study of the human taste region. Eur J Neurosci 11:2985-2988.

Friston KJ, Holmes AP, Poline JB, Grasby PJ, Williams SCR, Frackowiak RS, Turner R (1995) Analysis of fMRI time-series revisited. Neuroimage 2:45-53.

Friston KJ, Buechel C, Fink GR, Morris J, Rolls E, Dolan RJ (1997) Psychophysiological and modulatory interactions in neuroimaging. Neuroimage 6:218-229.

Friston KJ, Harrison L, Penny W (2003) Dynamic causal modelling. Neuroimage 19:1273-1302.

Friston KJ, Li B, Daunizeau J, Stephan KE (2011) Network discovery with DCM. Neuroimage 56:1202-1221.

Friston KJ, Litvak V, Oswal A, Razi A, Stephan KE, Van Wijk BC, Ziegler G, Zeidman P (2016) Bayesian model reduction and empirical Bayes for group (DCM) studies. Neuroimage 128:413-431.

Gitelman DR, Penny WD, Ashburner J, Friston KJ (2003) Modeling regional and psychophysiologic interactions in fMRI: the importance of hemodynamic deconvolution. Neuroimage 19:200-207.

Green BG, George P (2004) "Thermal taste" predicts higher responsiveness to chemical taste and flavor. Chem Senses 29:617-628.

Green BG, Shaffer GS, Gilmore MM (1993) Derivation and evaluation of a semantic scale of oral sensation magnitude with apparent ratio properties. Chem Senses 18:683-702.

Green BG, Dalton P, Cowart B, Shaffer G, Rankin K, Higgins J (1996) Evaluating the "labeled magnitude scale" for measuring sensations of taste and smell. Chem Senses 21:323-334.

Green BG, Alvarez-Reeves M, George P, Akirav C (2005) Chemesthesis and taste: evidence of independent processing of sensation intensity. Physiol Behav 86:526-537.

Hayes JE, Wallace MR, Knopik VS, Herbstman DM, Bartoshuk LM, Duffy VB (2011) Allelic variation in TAS2R bitter receptor genes associates with variation in sensations from and ingestive behaviors toward common bitter beverages in adults. Chem Senses 36:311-319.

Hayes JE, Feeney EL, Nolden AA, McGeary JE (2015) Quinine bitterness and grapefruit liking associate with allelic variants in TAS2R31. Chem Senses 40:437-443.

Henson RN, Price CJ, Rugg MD, Turner R, Friston KJ (2002) Detecting latency differences in event-related BOLD responses: application to words vs nonwords and initial vs repeated face presentations. Neuroimage 15:83-97.

Herrero M-T, Barcia C, Navarro J (2002) Functional anatomy of thalamus and basal ganglia. Childs Nerv Syst 18:386-404.

Hillyard SA, Vogel EK, Luck SJ (1998) Sensory gain control (amplification) as a mechanism of selective attention: electrophysiological and neuroimaging evidence. Philos Trans R Soc Lond B Biol Sci 353:1257-1270. 
Hutton C, Bork A, Josephs O, Deichmann R, Ashburner J, Turner R (2002) Image distortion correction in fMRI: a quantitative evaluation. Neuroimage 16:217-240.

Hutton C, Deichmann R, Turner R, Andersson JLR (2004) Combined correction for geometric distortion and its interaction with head motion in fMRI. Paper presented at International Society for Magnetic Resonance in Medicine Twelfth Scientific Meeting and Exhibition, Kyoto, Japan, May.

Jacobs R, Renken R, Aleman A, Cornelissen FW (2012) The amygdala, topdown effects, and selective attention to features. Neurosci Biobehav Rev 36:2069-2084

Katz DB, Nicolelis MA, Simon SA (2002) Gustatory processing is dynamic and distributed. Curr Opin Neurobiol 12:448-454.

Kauramäki J, Jääskeläinen IP, Sams M (2007) Selective attention increases both gain and feature selectivity of the human auditory cortex. PLoS One 2:e909.

Kim U-K, Breslin PAS, Reed D, Drayna D (2004) Genetics of human taste perception. J Dent Res 83:448-453.

Kosar E, Grill HJ, Norgren R (1986) Gustatory cortex in the rat. II. Thalamocortical projections. Brain Res 379:342-352.

Krettek JE, Price JL (1977) Projections from the amygdaloid complex to the cerebral cortex and thalamus in the rat and cat. J Comp Neurol 172:687722 .

Lim J, Urban L, Green BG (2008) Measures of individual differences in taste and creaminess perception. Chem Senses 33:493-501.

Macey PM, Macey KE, Kumar R, Harper RM (2004) A method for removal of global effects from fMRI time series. Neuroimage 22:360-366.

Meyerhof W, Batram C, Kuhn C, Brockhoff A, Chudoba E, Bufe B, Appendino G, Behrens M (2010) The molecular receptive ranges of human TAS2R bitter taste receptors. Chem Senses 35:157-170.

Morris JS, Friston KJ, Dolan RJ (1997) Neural responses to salient visual stimuli. Proc Biol Sci 264:769-775.

Morris JS, Öhman A, Dolan RJ (1999) A subcortical pathway to the right amygdala mediating "unseen" fear. Proc Natl Acad Sci U S A 96:16801685.

Kaskan PM, Dean AM, Nicholas MA, Mitz AR, Murray EA (2019) Gustatory responses in macaque monkeys revealed with fMRI: Comments on taste, taste preference, and internal state. NeuroImage 184:932-942.

Norgren R (1976) Taste pathways to hypothalamus and amygdala. J Comp Neurol 166:17-30.

Norgren R (1984) Central neural mechanisms of taste. In Handbook of Physiology-The Nervous System III. Sensory Processes 1., I. DarienSmith, ed. (Washington, DC: American Physiological Society), pp 10871128.

Norgren R (1990) Gustatory System. In The Human Nervous System, G. Paxinos, ed. (San Diego: Academic Press), pp 845-861.

Norgren R, Leonard CM (1971) Taste pathways in rat brainstem. Science 173:1136-1139.

O’Doherty J, Rolls ET, Francis S, Bowtell R, McGlone F (2001) Representation of pleasant and aversive taste in the human brain. J Neurophysiol 85:1315-1321.

Oldfield RC (1971) The assessment and analysis of handedness: the Edinburgh inventory. Neuropsychologia 9:97-113.

Pepino MY, Mennella JA (2007) Effects of cigarette smoking and family history of alcoholism on sweet taste perception and food cravings in women. Alcohol Clin Exp Res 31:1891-1899.

Price JL, Slotnick BM (1983) Dual olfactory representation in the rat thalamus: an anatomical and electrophysiological study. J Comp Neurol 215:63-77

Pritchard TC, Hamilton RB, Morse JR, Norgren R (1986) Projections of thalamic gustatory and lingual areas in the monkey, Macaca fascicularis. $\mathrm{J}$ Comp Neurol 244:213-228.

Pritchard TC, Hamilton RB, Norgren R (2000) Projections of the parabrachial nucleus in the old world monkey. Exp Neurol 165:101-117.

Samuelsen CL, Gardner MP, Fontanini A (2012) Effects of cue-triggered expectation on cortical processing of taste. Neuron 74:410-422.

Satterthwaite TD, Elliott MA, Gerraty RT, Ruparel K, Loughead J, Calkins ME, Eickhoff SB, Hakonarson H, Gur RC, Gur RE, Wolf DH (2013) An improved framework for confound regression and filtering for control of motion artifact in the preprocessing of resting-state functional connectivity data. Neuroimage 64:240-256.
Shi C-J, Cassell MD (1998) Cortical, thalamic, and amygdaloid connections of the anterior and posterior insular cortices. J Comp Neurol 399:440468

Small DM, Jones-Gotman M, Zatorre RJ, Petrides M, Evans AC (1997) A role for the right anterior temporal lobe in taste quality recognition. J Neurosci 17:5136-5142.

Small DM, Zatorre RJ, Jones-Gotman M (2001a) Changes in taste intensity perception following anterior temporal lobe removal in humans. Chem Senses 26:425-432.

Small DM, Zatorre RJ, Jones-Gotman M (2001b) Increased intensity perception of aversive taste following right anteromedial temporal lobe removal in humans. Brain 124:1566-1575.

Small DM, Gregory MD, Mak YE, Gitelman D, Mesulam MM, Parrish T (2003) Dissociation of neural representation of intensity and affective valuation in human gustation. Neuron 39:701-711.

Small DM, Voss J, Mak YE, Simmons KB, Parrish T, Gitelman D (2004) Experience-dependent neural integration of taste and smell in the human brain. J Neurophysiol 92:1892-1903.

Swanson LW, Petrovich GD (1998) What is the amygdala? Trends Neurosci 21:323-331.

Thibodeau M, Bajec M, Pickering G (2017) Orosensory responsiveness and alcohol behaviour. Physiol Behav 177:91-98.

Timbie C, Barbas H (2015) Pathways for emotions: specializations in the amygdalar, mediodorsal thalamic, and posterior orbitofrontal network. J Neurosci 35:11976-11987.

Todd N, Moeller S, Auerbach EJ, Yacoub E, Flandin G, Weiskopf N (2016) Evaluation of 2D multiband EPI imaging for high-resolution, wholebrain, task-based fMRI studies at 3T: sensitivity and slice leakage artifacts. Neuroimage 124:32-42.

Veldhuizen MG, Bender G, Constable RT, Small DM (2007) Trying to detect taste in a tasteless solution: modulation of early gustatory cortex by attention to taste. Chem Senses 32:569-581.

Veldhuizen MG, Shepard TG, Wang M-F, Marks LE (2010) Coactivation of gustatory and olfactory signals in flavor perception. Chem Senses 35:121-133.

Veldhuizen MG, Albrecht J, Zelano C, Boesveldt S, Breslin P, Lundström JN (2011a) Identification of human gustatory cortex by activation likelihood estimation. Hum Brain Mapp 32:2256-2266.

Veldhuizen MG, Douglas D, Aschenbrenner K, Gitelman DR, Small DM (2011b) The anterior insular cortex represents breaches of taste identity expectation. J Neurosci 31:14735-14744.

Vuilleumier P (2015) Affective and motivational control of vision. Curr Opin Neurol 28:29-35.

Wallroth R, Ohla K (2018) As soon as you taste it: evidence for sequential and parallel processing of gustatory information. eNeuro 5: ENEURO.0269-18.2018.

Wang L, Gillis-Smith S, Peng Y, Zhang J, Chen X, Salzman CD, Ryba NJP, Zuker CS (2018) The coding of valence and identity in the mammalian taste system. Nature 558:127-131.

Womelsdorf T, Fries P (2007) The role of neuronal synchronization in selective attention. Curr Opin Neurobiol 17:154-160.

Worsley KJ, Friston KJ (1995) Analysis of fMRI time-series revisited-again. Neuroimage 2:173-181

Yeung AWK, Tanabe HC, Suen JLK, Goto TK (2016) Taste intensity modulates effective connectivity from the insular cortex to the thalamus in humans. Neuroimage 135:214-222.

Yeung AWK, Goto TK, Leung WK (2017) Basic taste processing recruits bilateral anteroventral and middle dorsal insulae: an activation likelihood estimation meta-analysis of fMRI studies. Brain Behav 7:e00655.

Yokomukai Y, Cowart BJ, Beauchamp GK (1993) Individual differences in sensitivity to bitter-tasting substances. Chem Senses 18:669-681.

Zald DH, Pardo JV (2000) Cortical activation induced by intraoral stimulation with water in humans. Chem Senses 25:267-275

Zeidman P, Jafarian A, Corbin N, Seghier ML, Razi A, Price CJ, Friston KJ (2019a) A guide to group effective connectivity analysis, part 1: first level analysis with DCM for fMRI. Neuroimage 200:174-190.

Zeidman P, Jafarian A, Seghier ML, Litvak V, Cagnan H, Price CJ, Friston KJ (2019b) A guide to group effective connectivity analysis, part 2: second level analysis with PEB. Neuroimage 200:12-25. 